\title{
CONSERVATIVE POSTNATAL MANAGEMENT OF ANTENATALLY DIAGNOSED CONGENITAL PULMONARY AIRWAY MALFORMATIONS (CPAM)
}

\section{(i) ABSTRACT \& KEY WORDS:}

Aim: Management of Congenital Pulmonary Airway Malformations (CPAM) is controversial, especially for asymptomatic patients. We aim to describe the clinical manifestations and management of CPAM at a tertiary paediatric hospital using a retrospective audit. Method: Infants with CPAM were identified on the Fetal Diagnostic Unit database from 2007-2014. Information on antenatal and postnatal management was collected from medical record.

Results: Thirty-five infants with antenatally diagnosed CPAM were included. Fetal CPAM volume ratio (CVR) was calculated from antenatal ultrasound measurement and used to categorize the infants into 3 groups of large (CVR $\geq 1.6, n=8$ ), medium (CVR of $0.5-1.6, n=12$ ) and small CPAM (CVR of $\leq 0.5, n=15)$, respectively. Ten infants $(10 / 35=29 \%)$ were symptomatic in the neonatal period. Overall, nine infants (26\%) had surgical resection, among whom 8 had large or medium-sized CPAM lesions as defined by the antenatal CVR. Three infants had neonatal emergency surgery and the remaining 6 had late elective surgery. Histology of 8 cases showed CPAM, but 1 case showed congenital lobar emphysema. Criteria for surgery varied and included persistent symptoms after birth, complications during childhood and persistently abnormal chest X-ray. Most asymptomatic infants with CPAM were safely managed using a conservative approach, with no significant increase in late symptoms or complications.

Conclusions: Conservative management of CPAM may be considered for infants/children who remain asymptomatic, especially those with a small lesion. For large and medium-sized CPAM, delineation using CT is required, and surgery may be beneficial to prevent late symptoms and the risk of emergency surgery.

Key words: congenital pulmonary airway malformation, neonates, malformations lung, Respiratory Distress Syndrome, prenatal diagnosis

\section{Abbreviations:}

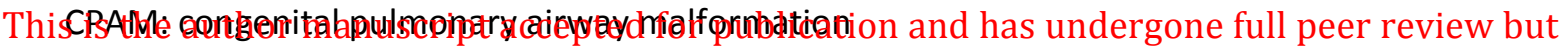

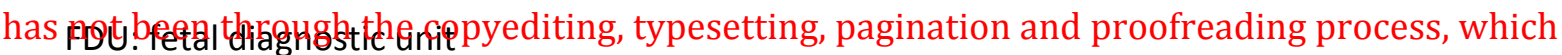
may lead to differences between this version and the Version of Record. Please cite this article
CVR: CAM volume ratio as doi: 10.1111/jpc.13727

This article is protected by copyright. All rights reserved. 
CXR: chest X-ray

US: ultrasound

SCN: special care nursery

NICU: neonatal intensive care unit

CLE: congenital lobar emphysema

This article is protected by copyright. All rights reserved. 


\section{(ii) TEXT}

\section{Introduction}

Congenital Pulmonary Airway Malformations (CPAM) is a rare abnormality of lung development characterized by excessive proliferation and cystic dilatation of terminal respiratory bronchioles. The incidence is approximately 1 in $25,000-35,000^{1}$, however this may be an under-representation due to the sonographer-dependent nature of diagnosis on antenatal ultrasound, and the limited access to testing in some populations.

Most congenital lung lesions including CPAM are first diagnosed during the routine second trimester morphology scan. CPAM has a variable natural history in-utero that may result in hydrops in up to $40 \%{ }^{2}$, or regression and resolution in $29 \%^{3}$. Perinatal mortality of antenatally diagnosed CPAM ranges from $9-49 \%{ }^{1,4}$. Adverse prognosis is associated with non-immune hydrops, mediastinal shift and pulmonary hypoplasia ${ }^{1}$. The clinical severity of CPAM is also related to its size and type, and the need for surgery ${ }^{5}$. A prognostic parameter known as the CAM volume ratio (CVR) has been developed to normalise the tumour volume for fetal head circumference, in order to standardize the tumour growth with gestational age. A CVR of $>1.6$ was associated with increased risk of hydrops ${ }^{2}$. Risk of hydrops for lesions with lower CVR and without a dominant cyst is $<3 \%^{2}$.

Postnatally, clinical presentations ranges from being asymptomatic to early respiratory failure ${ }^{4}$. For symptomatic infants with CPAM, early surgery with cystectomy or lobectomy is necessary. However for asymptomatic lesions, management is contentious. The two main approaches are conservative management (or "watch and wait" approach) and prophylactic surgical resection, which is further divided into the immediate versus delayed approach. Currently, there is no standard management guideline and therefore management often depends on the clinician's preference.

We have conducted a retrospective study on the course of disease and scope of management of CPAM diagnosed antenatally in a tertiary perinatal unit. We aim to review the current local practice, describe the disease spectrum, and provide a framework in the postnatal management of future CPAM cases.

This article is protected by copyright. All rights reserved. 


\section{Materials \& Method}

Cases of CPAM were identified on the Fetal Diagnostic Unit database at Monash Medical Centre in Melbourne, Australia, from 2007-2014. Monash Medical Centre has a neonatal intensive care unit with 4,000 deliveries per year and one of only three referral centres for CPAM in the state. During this period, 8 different pediatric surgeons had been on the on-call roster for consultation. Clinical information was retrieved from medical record. Imaging information about the CPAM lesion was gathered from the radiology database. Serial ultrasound scans performed to monitor the size and growth of CPAM lesions were reviewed for each pregnancy. Lesions subsequently reclassified on ultrasound as either bronchopulmonary sequestration or congenital lobar emphysema (CLE) were excluded from this study. Data was collected for each pregnancy, including maternal age, gravidity and parity, gestation at diagnosis, serial measurements on antenatal ultrasound, estimated fetal weight, amniotic fluid index and antenatal surgical reviews. Infant information collected included gender, gestation at delivery, apgar scores, resuscitation required, admission to special care nursery (SCN)/neonatal intensive care unit (NICU), clinical presentation, respiratory support, radiological findings, length of hospital stay, outpatient surgical review, development of symptoms/ complications, indication and age at CPAM resection, histopathology of resected lesions and age at discharge from surgical follow-up.

CVR was calculated based on fetal ultrasound reports as CVR $=$ (Length $X$ Height $X$ Width $X$ $0.52) /$ Head Circumference ${ }^{2}$. We calculated the CVR for serial fetal ultrasounds for all cases, starting from the first ultrasound usually performed between 23-28 weeks gestation, and the largest CVR value was used for each infant in subsequent analyses. We have divided the cases of CPAM according to their largest CVR value, into the categories of large (CVR $\geq 1.6)$, medium (CVR $0.5-1.5)$ and small lesions (CVR<0.5). The cutoff of CVR at 1.6 was used due to its correlation with increased risks of fetal hydrops ${ }^{2}$. Infants were classified based on the size of the CPAM lesion, the presence of symptoms at birth, inpatient versus outpatient follow up, and surgery or conservative management. Approval was obtained from the Monash Health human research and ethics committee for waiver of individual patient consent as the study involved retrospective collection, use and disclosure of data in a de-identified format. Histopathology results were available for all resected lesions; all cases were included in this study based on ultrasound findings consistent with CPAM.

This article is protected by copyright. All rights reserved. 


\section{Results}

Overview

During the 8-year period, there were a total of 41 cases of antenatally diagnosed CPAM. Six infants were excluded: 1 fetal death in-utero with the CPAM occupying the entire left hemithorax (CVR $=1.4), 4$ fetuses with CPAM deemed small enough to deliver safely at local regional hospitals, and 1 case with medium-sized CPAM not included due to incomplete medical records. The remaining 35 infants were included in this report. Large CPAM accounted for $23 \%(8 / 35)$ of cases, $34 \%$ were of medium size $(12 / 35)$ and the majority were of small size (43\%; 15/35) (Figure 1$)$.

All 35 infants had a chest X-ray (CXR) on day 1 of life and the paediatric surgeon on-call was consulted. Infants discharged without surgery were followed up in surgical outpatient clinic at 6 weeks of age with a repeat CXR. The subsequent duration of outpatient follow-up was variable, usually between 4 months to 2 years, with some infants still being followed-up at 4 years of age. Variation in duration of follow up was due to individual surgeon's preference and practice.

\section{Symptoms in the neonatal period}

Ten out of the 35 infants had respiratory symptoms during the neonatal period ranging from tachypnoea to requiring non-invasive ventilation via continuous positive airway pressure (CPAP). Of these infants, 4 had large lesions, 5 were medium-sized and 1 had small CPAM (Figure 1).

\section{Neonatal Surgery}

Respiratory symptoms resolved in 7 out of the 10 symptomatic infants without surgery. The remaining 3 infants were all born at term with large CPAM, required neonatal surgery, and were managed by 3 different pediatric surgeons. The first infant required CPAP support and $\mathrm{CT}$ scan at $2.5 \mathrm{wks}$ of life showed a left lower lobe CPAM. This infant remained dependent on CPAP support. A left lower lobectomy was carried out at 2 months of age and the histology demonstrated CLE instead. The infant was discharged from follow up at 4 years of age.

In the second case of neonatal surgery, the infant required CPAP from birth. CT demonstrated right-sided CPAM with mediastinal shift. Right lower lobectomy was

This article is protected by copyright. All rights reserved. 
performed on day 19 with postoperative pneumothorax. Histology was consistent with type I CPAM. Her total length of inpatient stay was 31 days. She has been discharged from follow up at 1 year of age.

The third case was an infant who was tachypnoeic without needing respiratory support or oxygen. CT on day 5 showed a large right upper lobe CPAM. Parents opted for elective resection of the lesion on day 8 before flying home interstate. Histopathology was consistent with CPAM with multiple variable sized irregular cysts. The patient was discharged on day 22.

\section{Surgery in infancy and early childhood}

Six infants had elective resection of CPAM during infancy and childhood, of whom 1 infant had large CPAM, 4 with medium-sized CPAM and 1 with a small lesion. Three of these 6 infants had symptomatic complications of CPAM prior to surgery (see below).

In the group of infants with large CPAM lesions who were asymptomatic during the neonatal period $(n=4), 3$ had abnormal CXR of which 1 went on to have a lobectomy at 2 months of age, after a CT showing large left CPAM and mediastinal shift. Histology confirmed the diagnosis of type II CPAM.

Of infants with medium-sized CPAM, 2 of the 5 infants with resolved neonatal symptoms and abnormal CXR had lobectomy for late complications. One patient had recurrent infection with lobectomy at 6 months. The other presented with failure to thrive with lobectomy at 9 months. Histology showed type II CPAM in both cases.

Elective surgery was performed in 2 infants with medium-sized CPAM who were asymptomatic in the neonatal period with normal CXR. The first infant developed abnormality of CXR at subsequent review and elective lobectomy was performed at 4 years of age. Histopathology showed a unilocular cyst with multiple cystic spaces in adjacent lung parenchyma. The other infant developed an infection of the CPAM and had left lower lobectomy at 18 months of age. Histopathology was consistent with CPAM.

The final case of elective lobectomy occurred in an asymptomatic infant with a small CPAM with normal CXR. The infant was followed up at 6 weeks of age and the surgical team 
requested a CT due to a single cystic CPAM on antenatal scan, believed to be associated with malignant transformation. The lobectomy was performed at 10 months of age and histopathology was consistent with type I CPAM with a 20x22mm cyst.

Overall, 3 of the 6 infants offered elective surgery had clinical symptoms during infancy. One surgeon was responsible for 4 of the 6 elective surgeries, including 2 infants with symptoms and 2 without symptoms.

Notably, there was 1 infant who developed infected CPAM at 2.5 years of age but not offered surgery - the infant had large CPAM and was initially asymptomatic. The plan is to excise the CPAM only if another infection occurs.

\section{Infants with neonatal respiratory symptoms that resolved}

There were 7 infants with neonatal respiratory symptoms that resolved, and all of them had abnormal CXR at birth. Two of these infants, both with medium-sized lesions, had surgery at 6 and 9 months of age respectively after developing clinical symptoms (as discussed above).

\section{Asymptomatic infants}

Of the 4 asymptomatic infants with large CPAM, 3 had abnormal CXRs on day 1 of life. One was offered elective surgery as discussed above. Two are currently being reviewed annually (aged $2 \& 4$, respectively), though their CXR have both normalized. The infant with normal CXR at birth developed infected CPAM at 2.5 years of age, but has been managed conservatively (discussed above).

Seven infants with medium-sized lesions were asymptomatic at birth, 5 of whom had abnormal CXR at birth. All five are being conservatively managed. Notably, 2 patients still have persistent CXR abnormalities (age $3 \& 4$ years respectively). The 2 other infants with no neonatal symptoms had normal CXR. They both had late lobectomy, of which 1 was due to infected CPAM (see section on 'Surgery in infancy and childhood').

Of the 14 asymptomatic infants with small lesions, 4 had abnormal CXR. Two are currently followed-up and 2 failed to attend outpatient appointments. Of the infants with normal CXR ( $n=10), 1$ had lobectomy at 10 months of age despite being asymptomatic (discussed above), 7 were conservatively managed and 2 patients failed to attend outpatient appointments.

This article is protected by copyright. All rights reserved. 


\section{Discussion}

Management of CPAM varies depending on individual clinical practice. Approximately 30$40 \%$ of infants with CPAM are symptomatic in the neonatal period ${ }^{6}$, which is similar to our result $(29 \%, 10 / 35)$ (Table 1$)$. One study reported $86 \%(13 / 14,1$ died) of symptomatic infants with CPAM required surgery due to significant respiratory distress, at a mean age of 1 month (0-9 years) ${ }^{6}$. This high severity of respiratory problems and rate of surgery was not reflected in our cohort. Our cohort had no postnatal mortality, and of the 10 infants with neonatal symptoms, $5(50 \%)$ had surgery either during the neonatal period $(n=3,1$ of which was found to be CLE) or infancy $(n=2)$. This may be related to our relatively conservative approach where neonatal surgery is usually only offered for persistent clinical symptoms. This potentially led to a slightly higher rate of late complications during infancy and childhood $(13 \%, 4 / 32)$. Our overall surgery rate is $26 \%$ ( $9 \%$ neonatal; $17 \%$ infancy/early childhood) (Table 1). Our study focuses on cases diagnosed antenatally as CPAM on serial ultrasound. Histology of 8 out of the 9 resected lesions demonstrated CPAM. It is still possible that some of the conservatively managed cases were of other histopathologies.

\section{Symptom development (early vs. late)}

Infants and children with CPAM may develop symptoms including respiratory distress, chronic cough, poor feeding or respiratory failure, which are indications for surgery ${ }^{4}$. One study suggested that a large number of initially asymptomatic infants (18/21) would later develop symptoms due to the CPAM in early childhood ${ }^{6}$. However, in this paper, 13 of these 18 infants/children (i.e. 72\%) were only diagnosed postnatally after the development of symptoms, in contrast to our cohort, which only included patients with antenatally diagnosed CPAM.

A systematic review of all congenital cystic lung lesions (including CPAM, bronchopulmonary sequestration and hybrid lesions) suggested that only $3-10 \%$ of infants diagnosed antenatally would develop postnatal symptoms ${ }^{3}$. Unfortunately, data specific to CPAM lesions was not separately reported. Our data suggested a slightly higher incidence of late symptom development with CPAM; where 4/32 (13\%) infants who did not have neonatal surgery developed late symptoms, with 3 of these children going on to have surgery.

This article is protected by copyright. All rights reserved. 


\section{Prophylactic surgical excision of CPAM}

While some clinicians recommend surgery only after the development of symptoms ${ }^{7,8}$, other reports advocate early, prophylactic surgical excision regardless of the presence of symptoms ${ }^{9}$, to reduce the risk of malignancy ${ }^{10}$, facilitate optimal lung growth and minimise the risk of pulmonary hypoplasia, infections and pneumothorax ${ }^{11}$. One study ${ }^{11}$ reported 17 out of 29 asymptomatic infants with CPAM had prophylactic surgery, with the majority of these infants $(n=14)$ having large $(>3 \mathrm{~cm})$ or fluid filled lesions. Again, our cohort was managed more conservatively, and 3 of the $23(13 \%)$ patients who never developed any symptoms received prophylactic surgery.

\section{Timing of elective surgery}

Some centers propose surgery in the neonatal period ${ }^{12}$, while others consider the risk of anesthesia and the length of postoperative ventilation to be unjustified ${ }^{13}$, and therefore surgery should be delayed to 6 months ${ }^{14}$ to 2 years ${ }^{15}$. On the other hand, one study reported no difference in complications between immediate and delayed surgery ${ }^{16}$. However, a greater than 2-fold increase in risk of complications has been reported following emergency surgery in symptomatic patients compared to elective surgery ${ }^{3}$, thus strengthening the argument for early elective surgery.

\section{Conservative management}

In contrast, those who advocate conservative management suggest that the risk of malignancy in CPAM is poorly defined ${ }^{17}$ and influence on lung growth is small, therefore continued review is appropriate in asymptomatic patients ${ }^{7}$. Our centre has largely adopted the conservative approach to asymptomatic CPAM, with only few (13\%) being offered prophylactic surgery. Importantly, this did not significantly increase our rate of late symptoms/complications. Nevertheless, being a retrospective study, our result is limited by the variable and relatively short follow-up periods. Future studies are required with larger sample sizes and longer follow-up to evaluate the conservative approach.

\section{$\underline{\text { Radiological investigations }}$}

Antenatal ultrasound findings of CPAM are not predictive of postnatal course $\mathrm{e}^{12}$, and neonatal CXRs has a high false-negative rate for CPAM $^{1}$. Therefore, some suggest that all infants with antenatally diagnosed CPAM should receive a postnatal CT scan ${ }^{4}$. The balance between appropriately investigating a potentially significant lung lesion versus unnecessary 
exposure to radiation in childhood should be considered. Furthermore, many asymptomatic lesions may not be resected immediately following $\mathrm{CT}$ scan and therefore may require repeated radiological monitoring and radiation exposure.

In our cohort, infants with large CPAM diagnosed on antenatal ultrasound had higher rates of symptoms (63\%) and surgery (50\%) (Table 1). Our result suggests that it is appropriate for asymptomatic infants with antenatally diagnosed large or medium-sized CPAM to have a postnatal CT scan in the first few months of life to delineate the lesion. Small lesions were less likely to become symptomatic and therefore should be investigated on a case-by-case basis, with CT scans if symptoms appear and/or CXR abnormalities persist.

\section{Conclusion}

Overall, a conservative approach was used in our cohort with relatively low rates of surgery and few complications. Conservative management may benefit patients by minimizing the risks of unnecessary surgery. However, the long period of outpatient observation and associated dropout in outpatient attendance, increased radiographic investigations and radiation exposure, as well as the increased risks of emergency surgery for late complications must be taken into consideration as potential issues with the conservative approach. The conservative approach is appropriate if an infant remains asymptomatic especially with a small lesion. For large and medium-sized CPAM evaluated on CT scan, surgery may be beneficial to prevent late symptoms and the risk of emergency surgery.

This article is protected by copyright. All rights reserved. 


\section{(iii) ACKNOWLEDGEMENTS:}

The project is supported by the Victorian Government's Operational Infrastructure Support Program. FYW is supported by the NHMRC Career Development Fellowship.

This article is protected by copyright. All rights reserved. 


\section{(iv) REFERENCES:}

1. Laberge J, Flageole H, Pugash D, Khalife S, Blair G, Filiatrault D, et al. Outcome of the Prenatally Diagnosed Congenital Cystic Adenomatoid Malformation: A Canadian Experience. Fetal Diagn Ther. 2001;16:178-86.

2. Crombleholme TM, Coleman B, Hedrick H, Liechty K, Howell L, Flake A, et al. Cystic adenomatoid malformation volume ratio predicts outcome in prenatally diagnosed cystic adenomatoid malformation of the lung. J Pediatr Surg. 2002;37(3):331-8.

3. Stanton M, Njere I, Ade-Ajayi N, Patel S, Davenport M. Systematic review and metaanalysis of the postnatal management of congenital cystic lung lesions. J Pediatr Surg. 2009;44(5):1027-33.

4. Di Prima F, Bellia A, Inclimona G, Grasso F, Teresa M, Nazario Cassaro M. Antenatally diagnosed congenital cystic adenomatoid malformations (CCAM): Research Review. J Prenat Med. 2012;6(2):22-30.

5. Sfakianaki A, Copel J. Congenital Cystic Lesions of the Lung: Congenital Cystic Adenomatoid Malformation and Bronchopulmonary Sequestration. Rev Obstet Gynecol. 2012;5(2):85-93.

6. Wong A, Vieten D, Singh S, Harvey JG, Holland AJ. Long-term outcome of asymptomatic patients with congenital cystic adenomatoid malformation. Pediatr Surg Int. 2009;25(6):479-85.

7. Butterworth S, Blair G. Postnatal spontaneous resolution of congenital cystic adenomatoid malformations. J Pediatr Surg. 2005;40:832-24.

8. Aziz D, Langer JC, Tuuha SE, Ryan G, Ein SH, Kim PCW. Perinatally diagnosed asymptomatic congenital cystic adenomatoid malformation: to resect or not? J Pediatr Surg. 2004;39(3):329-34.

9. Parikh D, Samuel M. Congenital cystic lung lesions: is surgical resection essential? Pediatr Pulmonol. 2005;40(6):533-7.

10. West D, Nicholson AG, Colquhoun I, Pollock J. Bronchioloalveolar carcinoma in congenital cystic adenomatoid malformation of lung. Ann Thorac Surg. 2007;82(2):687-9. 11. Sauvat F, Michel JL, Benachi A, Emond S, Revillon Y. Management of asymptomatic neonatal cystic adenomatoid malformations. J Pediatr Surg. 2003;38(4):548-52.

12. Waszak $\mathrm{P}$, Claris $\mathrm{O}$, Lapillonne A. Cystic adenomatoid malformation of the lung: neonatal management of 21 cases. Pediatr Surg Int. 1999;15:326-31.

13. Adzick N, Flake A, Crombleholme TM. Management of congenital lung lesions. Semin Pediatr Surg. 2003;12(1):10-6.

This article is protected by copyright. All rights reserved. 
14. Laberge J, Puligandla P, Flageole H. Asymptomatic congenital lung malformations. Semin Pediatr Surg. 2005;14:16-33.

15. Papagiannopoulos K, Hughes S, Nicholson A. Cystic lung lesions in the paediatric and adult populations: surgical experience at the Brompton Hospital. Ann Thorac Surg. 2002;73:1594-8.

16. Colon N, Schlegel C, Pietsch J, Chung DH, Jackson GP. Congenital lung anomalies: can we postpone resection? J Pediatr Surg. 2012;47(1):87-92.

17. Priest JR, Williams GM, Hill DA, Dehner LP, Jaffe A. Pulmonary cysts in early childhood and the risk of malignancy. Pediatr Pulmonol. 2009;44(1):14-30.

This article is protected by copyright. All rights reserved. 
(v) TABLE 1:

Table 1: The incidence of clinical symptoms and surgery in infants with CPAM, categorised according to the fetal CAM volume ratio (CVR).

\begin{tabular}{|c|c|c|c|c|c|c|c|}
\hline \multirow{2}{*}{$\begin{array}{l}\text { CPAM Size } \\
\quad(C V R)\end{array}$} & \multirow{2}{*}{$\mathrm{n}$} & \multicolumn{3}{|c|}{ Symptomatic [n(\%)] } & \multicolumn{3}{|c|}{ Surgery $[n(\%)]$} \\
\hline & & Neonatal & Late & Total & Neonatal & Late & Total \\
\hline Large ( $\geq 1.6)$ & 8 & $4(50 \%)$ & $1^{\dagger}(13 \%)$ & $5(63 \%)$ & $3^{\#}(38 \%)$ & $1^{\ddagger}(13 \%)$ & $4(50 \%)$ \\
\hline $\begin{array}{c}\text { Medium (0.5- } \\
1.5) \\
\end{array}$ & 12 & $5(42 \%)$ & $\begin{array}{l}1\left(+2^{\S}\right) \\
(25 \%)\end{array}$ & $6(50 \%)$ & 0 & $\begin{array}{c}3+1^{\ddagger} \\
(33 \%)\end{array}$ & $4(33 \%)$ \\
\hline Small $(<0.5)$ & 15 & $1(7 \%)$ & 0 & $1(7 \%)$ & 0 & $1^{\ddagger}(7 \%)$ & $1(7 \%)$ \\
\hline Total & 35 & 10 (29\%) & $\begin{array}{l}2\left(+2^{5}\right) \\
(13 \%)^{9}\end{array}$ & $\begin{array}{c}12 \\
(34 \%)\end{array}$ & $3(9 \%)$ & $6(17 \%)$ & $9(26 \%)$ \\
\hline
\end{tabular}

Footnote: Symbols represent the following:

+ Not offered surg

$\ddagger$ asymptomatic

\# One infant had congenital lobar emphysema on histology

$\S$ These 2 infants also had neonatal symptoms

If Denominator is $n=32$, as 3 patients already had neonatal surgery 


\section{(vi) FIGURE LEGEND}

Figure 1: Flowchart representing all cases of CPAM between 2007 and 2014 managed at Monash Medical Centre, Victoria.

- Computer Tomography \& resection of CPAM

- Conservative Management

- Failure to attend outpatient appointment

CPAM - congenital pulmonary airway malformation

CVR - CAM volume ratio (Lesion size is as measured on last antenatal scan on record prior to delivery.)

Symptomic infants included those presenting with neonatal respiratory distress +/- need for respiratory support.

CXR - chest X-ray

Abn - abnormal

Tx - treatment of surgical resection

$\mathrm{CT}$ - computer tomography

Cons $\mathrm{Mx}$ - conservative management

FTA - failed to attend outpatient appointment

\# One case was postnatally diagnosed as congenital lobar emphysema on histology

This article is protected by copyright. All rights reserved. 


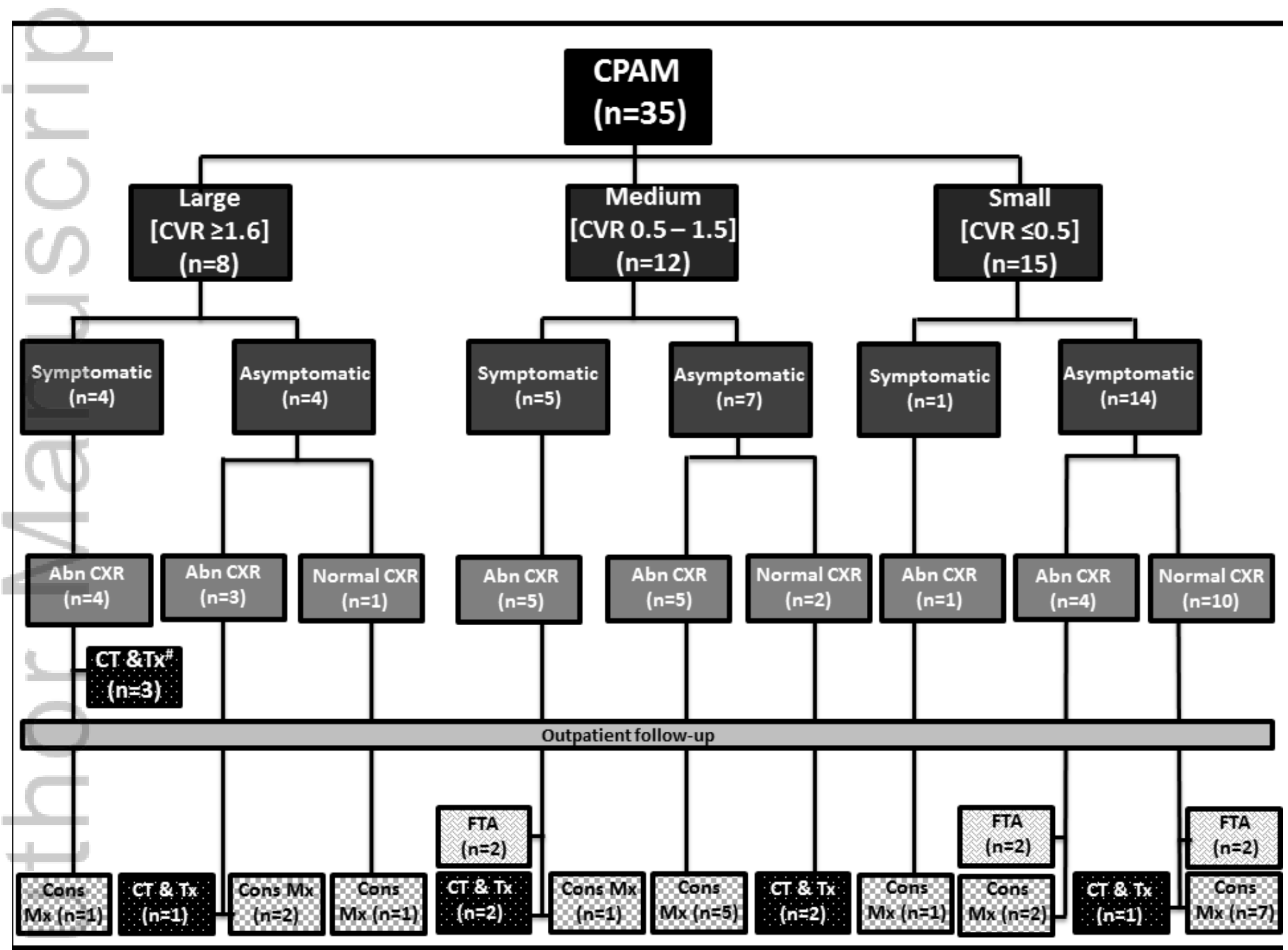

This article is protected by copyright. All rights reserved. 
Six points

What is already known on this topic:

1. Congenital Pulmonary Airway Malformations (CPAM) is usually identified on antenatal morphology ultrasound scan and has a wide clinical spectrum of symptoms and severity.

2. Using the fetal CAM volume ratio (CVR) to determine size of lesions can risk- stratify patients to predict prognosis.

3. Many paediatric surgical centres electively resect CPAM lesions due to their possible risk of recurrent infection, symptom development and malignant transformation.

\section{What this paper adds:}

1. Using a conservative approach, half of the infants with antenatally diagnosed CPAM and neonatal respiratory symptoms require surgery during the neonatal period or infancy.

2. Conservative management of asymptomatic infants with CPAM is feasible without increase in late complications, especially those with a small lesion.

3. Large and medium-sized CPAM have increased risks of symptom development, and should be evaluated by CT for consideration of prophylactic surgery to prevent late complications and the risk of emergency surgery.

This article is protected by copyright. All rights reserved. 


\section{University Library}

\section{- M M I N E R VA A gateway to Melbourne's research publications}

Minerva Access is the Institutional Repository of The University of Melbourne

Author/s:

Makhijani, AV;Wong, FY

Title:

Conservative post-natal management of antenatally diagnosed congenital pulmonary airway malformations.

\section{Date:}

2018-03

\section{Citation:}

Makhijani, A. V. \& Wong, F. Y. (2018). Conservative post-natal management of antenatally diagnosed congenital pulmonary airway malformations.. J Paediatr Child Health, 54 (3), pp.267-271. https://doi.org/10.1111/jpc.13727.

Persistent Link:

http://hdl.handle.net/11343/293513 